\title{
PSMA-SPECIFIC CAR-ENGINEERED $T$ CELLS FOR PROSTATE CANCER: CD28 OUTPERFORMS COMBINED CD28-41BB “SUPER- STIMULATION".
}

\author{
Gaia Zuccolotto ${ }^{*}$, Alessandro Penna ${ }^{2,}$, Giulio Fracasso ${ }^{3}$, Isabella Monia Montagner ${ }^{2,}$, Debora \\ Carpanese $^{2}$, Anna Tosi ${ }^{1}$ and Antonio Rosato ${ }^{1,2, *}$ \\ 1 Department of Surgery, Oncology and Gastroenterology, University of Padua, 35128 Padua, Italy \\ antonio.rosato@unipd.it (A.R.) gaia.zuccolotto@unipd.it (G.Z.) \\ 2 Veneto Institute of Oncology IOV - IRCCS, 35128 Padua, Italy; isabellamonia.montagner@iov.veneto.it (I.M.M.); \\ alessandro.penna.1994@gmail.com (A.P.); debora.carpanese@gmail.com (D.C.); \\ 3 Department of Medicine, University of Verona, 37134 Verona, Italy; giulio.fracasso@univr.it (G.F.) \\ * Correspondence: gaia.zuccolotto@unipd.it (G.Z.); antonio.rosato@unipd.it (A.R.)
}

\begin{abstract}
Despite advances in the understanding of its molecular pathophysiology, prostate cancer remains largely incurable, highlighting the need for novel therapies. We developed a chimeric antigen receptor (CAR) specific for prostate specific membrane antigen (PSMA), a glycoprotein that is overexpressed in prostate cancer, which expression involves neovasculature of several tumor entities, thus envisaging an additional antiangiogenic effect. To optimize the CAR design, we compared two CARs with signaling domains containing one or two T cell costimulatory elements, in addition to $\mathrm{CD} 3 \zeta$. Conversely, what has been described for other CARs, a third-generation CAR (containing CD28 and 41BB co-signaling domains) induced a potent antitumor effect similar to a second-generation CAR (containing CD28 co-signaling domain), though we observed a detrimental effect of the additional costimulatory domain that was attributed to increased activation-induced cell death (AICD). This "super-stimulation" resulted in exhaustion of cells, higher frequencies of cell death and, more importantly, the impossibility of sufficiently expanding the CAR cells to obtain the minimum number of cells requested for in vivo therapies. While the superiority of 2 nd and 3rd generation over 1st generation CAR T cells has been clearly shown in both preclinical and clinical studies, the optimal combination of costimulatory domains for 3rd generation CAR-T cells must still be defined and should be evaluated case-by-case in order to fine-tune immunotherapy approaches.
\end{abstract}

Keywords: Cancer immunotherapy; Prostate Cancer; CAR-T; PSMA.

\section{Introduction}

Adoptive cell transfer (ACT), utilizing autologous $\mathrm{T}$ cells engineered to express chimeric antigen receptors (CAR), has proven to be a highly effective strategy for the management of patients with relapsed or refractory B-cell malignancies [1-3]. However, the field remains in its infancy and these great strides are yet to be replicated in patients with advanced solid tumors [4-6]. In the context of Prostate Cancer (PCa), one of the most suitable targets is prostate specific membrane antigen (PSMA), which is a well-known tumor-associated antigen. In particular, PSMA expression levels 
differentiate normal and cancerous prostatic tissues. Interestingly, PSMA expression involves neovasculature of several tumor entities, thus envisaging an additional antiangiogenic effect. In this scenario, PSMA can represent a suitable target; indeed, it is currently exploited for both imaging and therapeutic purposes [7]. In CAR-based therapies, even though identifying valid targets to achieve effective tumor rejection while ensuring patient safety is an essential goal, it is well accepted that anti-tumor efficacy of adoptively transferred $\mathrm{T}$ cells requires efficient expansion and persistence in vivo [8-10]. Moreover, there is currently little understanding of how CAR structure impacts on these properties. Meanwhile, human T cells expressing CARs not infrequently mediate potent in vitro cytolysis, but show limited expansion, persistence, and anti-tumor efficacy [11-14]. Much work remains to be undertaken to more fully appreciate how CAR structure determines function and delineates the complexity of CAR intracellular signaling, as well the web of interactions between CAR $\mathrm{T}$ cells and other protagonist cells within the tumor microenvironment (TME) in vivo. Considerable effort continues to be applied to the optimization of the CAR construct itself to enhance antitumor potency, metabolism, proliferative capacity, and persistence $[15,16]$. It is becoming increasingly apparent that subtle differences in CAR design can have amplified effects both in vitro and - particularly - in vivo and that the optimal selection of CAR's extracellular targeting moiety, hinge, spacer, transmembrane domain (TMD), and intracellular costimulatory domain(s) (ICD), is crucial. Co-stimulation provided by a CD28 signaling domain in a second-generation CAR (2GCAR) substantially improved the efficacy of redirected $T$ cells in an antitumor attack, while other co-stimuli differentially modulate the T-cell effector functions in a specific fashion [17]. Combined co-stimulation by the so-called third-generation CAR (3G-CAR) with early CD28 and late costimulation by OX40 or 4-1BB promotes T-effector memory cell differentiation and protects cells from apoptosis [18]. In view of all these findings, understanding how the CAR structure influences the behavior of $\mathrm{T}$ cells is extremely relevant. Moreover, while the superiority of $2 \mathrm{nd}$ and $3 \mathrm{rd}$ generation over 1st generation CAR T cells has been clearly shown in both pre-clinical and clinical studies, the optimal combination of co-stimulatory domains for 3rd generation CAR-T cells remains to be defined and should be evaluated case-by-case in order to fine-tune immunotherapy approaches. With a view to of identifying the best experimental conditions able to ameliorate the biological properties of CAR-T cells in humans and, thus, to optimize clinical results of CAR T-cell therapy in patients with PCa, we designed and tested a 2 G and 3G CAR direct against the PSMA. In this work, the direct comparison of the efficacy of CAR constructs targeting PSMA indicates that a 2G CAR containing the CD28 co-stimulation domain is equally effective as a 3G CAR, which contains both CD28 and 4-1BB domains. However, most importantly, we confirmed what was observed in other works $[19,20]$, namely, that the effect of additional signaling modules is not additive, as often affirmed [21-23], and can in fact be detrimental. In particular, in this work we observed that the detrimental effect of the additional co-stimulatory domain was attributed to increased activation-induced cell death in T cells expressing the 3G CAR, as a result of augmented FasL expression that induces an exhaustion status represented by low proliferative and cytokine producing capacities, high rates of apoptosis, high expression of inhibitory receptors, and a reduced expansion of CAR T cells. Exhaustion has a significant role in limiting CAR efficacy and how CAR structural design affects the development of exhaustion has not been yet clearly understood. Here we demonstrate that CAR structure has a central role in predisposing CAR T cells to exhaustion, but above all we show how combined CD28-41BB "super-stimulation" can be counter-productive. 


\section{Materials and Methods}

\subsection{Cell Lines}

LNCaP, PC3 (both human prostate carcinoma cell lines) and 293T (human embryonic kidney cell line) were obtained from the American Type Culture Collection (ATCC). PC3-PSMA, a PC3 derivative cell line stably expressing human PSMA, has been previously described [24]. LNCaP, PC3, and PC3-PSMA, were cultured in RPMI 1640 medium, while DMEM medium was used for 293T; all cell lines were enriched with $10 \%$ heat-inactivated fetal bovine serum (FBS, Gibco). All media were supplemented with $2 \mathrm{mM}$ L-Glutamine, $100 \mathrm{U} / \mathrm{mL}$ penicillin, and $100 \mu \mathrm{g} / \mathrm{mL}$ streptomycin (all from Lonza) and maintained at $37^{\circ} \mathrm{C}$ in a humidified $5 \% \mathrm{CO}_{2}$ incubator. Firefly luciferase (fluc)-expressing PC3 and PC3-PSMA cell derivatives were obtained by viral transduction as previously described [25].

\subsection{T Cell Transduction}

The anti-PSMA CAR/eGFP lentiviral transfer vector (LV) and viral particle production in 293 T cells have been previously described [25]. To generate CAR-T cells, PBMC from healthy donors were activated 48 hours with OKT-3 (50 ng/mL; Ortho Biotech Inc) and human IL-2 (hIL-2, 300 U/mL; Proleukin; Novartis Pharmaceuticals). T cells were then infected with the viral supernatant for 18 hours at $37^{\circ} \mathrm{C}$ and $5 \% \mathrm{CO}_{2}$, in the presence of protamine sulfate $(40 \mathrm{mg} / \mathrm{mL}$; Sigma- Aldrich) and hIL-2 $(500 \mathrm{U} / \mathrm{mL})$. The supernatant was then changed with fresh complete medium containing hIL$2(100 \mathrm{U} / \mathrm{mL})$. Seventy-two hours later, PBMC were analyzed for CAR and eGFP expression. CART cells were re-stimulated once a week with irradiated (60 Gy) PC3-PSMA at a 10:1 ratio. Complete medium with fresh IL-2 was replenished twice a week.

\subsection{Phenotypic analysis}

All samples were analyzed with a FACSCalibur (BD Bioscience) and data were analyzed using FlowJo software. PSMA CARs were detected with the anti-c-myc mAb (clone 9E10; Sigma-Aldrich) or the isotype control (mouse IgG1, Southern Biotech, Milan, Italy), followed by a secondary antibody (PE-conjugated goat anti-mouse IgG; Southern Biotech). T cell phenotype was evaluated via: CD62L, CCR7, CD27, CD28, CD57 (eBioscience), Annexin V (BD Bioscience), FasL (BD Bioscience), PD-1 (eBioscience), TIM-3 (eBioscience), LAG-3 (eBioscience) and the relative isotype controls purchased from the same companies. All FACS plots presenting CAR T cell phenotype data were conducted on gated CAR ${ }^{+}$cells.

\subsection{Cytotoxicity assay}

The cytotoxic activity of $2 \mathrm{G}$ and $3 \mathrm{G}$ CAR T cells was assessed in a standard $4 \mathrm{~h}{ }^{51} \mathrm{Cr}$-release assay as previously reported [25]. PC3-PSMA, LNCaP and PC3 were used as target cells.

\subsection{Cytokine release assay}

To evaluate IFN- $\gamma$, IL- 2 and TNF- $\alpha$ production, an ELISA IFN- $\gamma$, IL-2 or TNF- $\alpha$ Screening Set (Thermo Scientific) was used, according to manufacturer's instructions. Briefly, $1 \times 10^{6}$ CAR T cells were seeded with $1 \times 10^{6}$ target cells (PC3 or PC3-PSMA) in triplicate wells in 96-well round bottom plates. Cytokine secretion was measured after 12 hours of incubation. Negative and positive controls were represented by CAR T cells unstimulated or treated with $40 \mathrm{ng} / \mathrm{mL}$ of PMA and $4 \mathrm{mg} / \mathrm{mL}$ of Ionomycin (Sigma-Aldrich), respectively. Supernatants were then analyzed on a VICTOR X4 (PerkinElmer). 


\subsection{Statistics}

Statistical analysis was performed by Student's $t$ test when only two value sets were compared. One-way ANOVA was used when the data involved three groups. Histograms represent mean values \pm standard deviation. $\mathrm{P}<0.05, \mathrm{P}<0.01$ or $\mathrm{P}<0.001$ were considered statistically significant and indicated by ${ }^{*}{ }^{* *}$ or ${ }^{* * *}$, respectively. Statistical analysis was performed using GraphPad Prism 7.0 software.

\section{Results}

\subsection{Construction and development of an efficient bidirectional LV for anti-PSMA 2G and 3G CAR expression}

The $2 \mathrm{G}$ and 3G CAR sequences were inserted into a LV carrying a bidirectional promoter [25] that allowed transcription of a reporter gene (eGFP) from the upstream minimal promoter minCMV, without affecting downstream expression of the anti-PSMA CAR from the efficient hPGK promoter (Fig. 1A). A 2G, and 3G CAR sequences, were designed and encoded the following components: the single-chain variable fragment ( $\mathrm{scFv}$ ) of the anti-PSMA antibody IgGD2B [26], a myc-tag for the cytofluorimetric detection, and the CD28 co-stimulatory molecule linked directly to the CD3 sequence for the 2G CAR; instead, the 3G CAR presents a second co-stimulatory molecule within the $\mathrm{CD} 28$ and $\mathrm{CD} 3 \zeta$ domains (Fig. 1B). We decided to exploit the anti-PSMA scFv (scFvD2B) for the construction of our CAR, because of its very appealing characteristics, in particular the nanomolar affinity for the target that is similar to the one evidenced by the whole J591 antibody [26]; the 2G CAR was already described from our group [25] and, encouraged from its high and specific lytic activity, we developed the 3G CAR to improve the activity and survival in vivo of the T cells transduced. T cells were transduced with LV 2G and 3G CAR PSMA/eGFP. In particular, one month after transduction, CAR-expressing cells were more than $90 \%$ when transduced with LV CAR PSMA/eGFP, as demonstrated by the high percentage of $\mathrm{CAR}^{+}$cells detected by flow cytometry analysis (Fig. 1C). Notably, the bidirectional LV vectors appeared to drive the expression of both the CAR ( $2 \mathrm{G}$ and $3 \mathrm{G}$ ) and the reporter gene with a very balanced efficiency, as demonstrated by the high intensity of eGFP signal (Fig. 1D). 


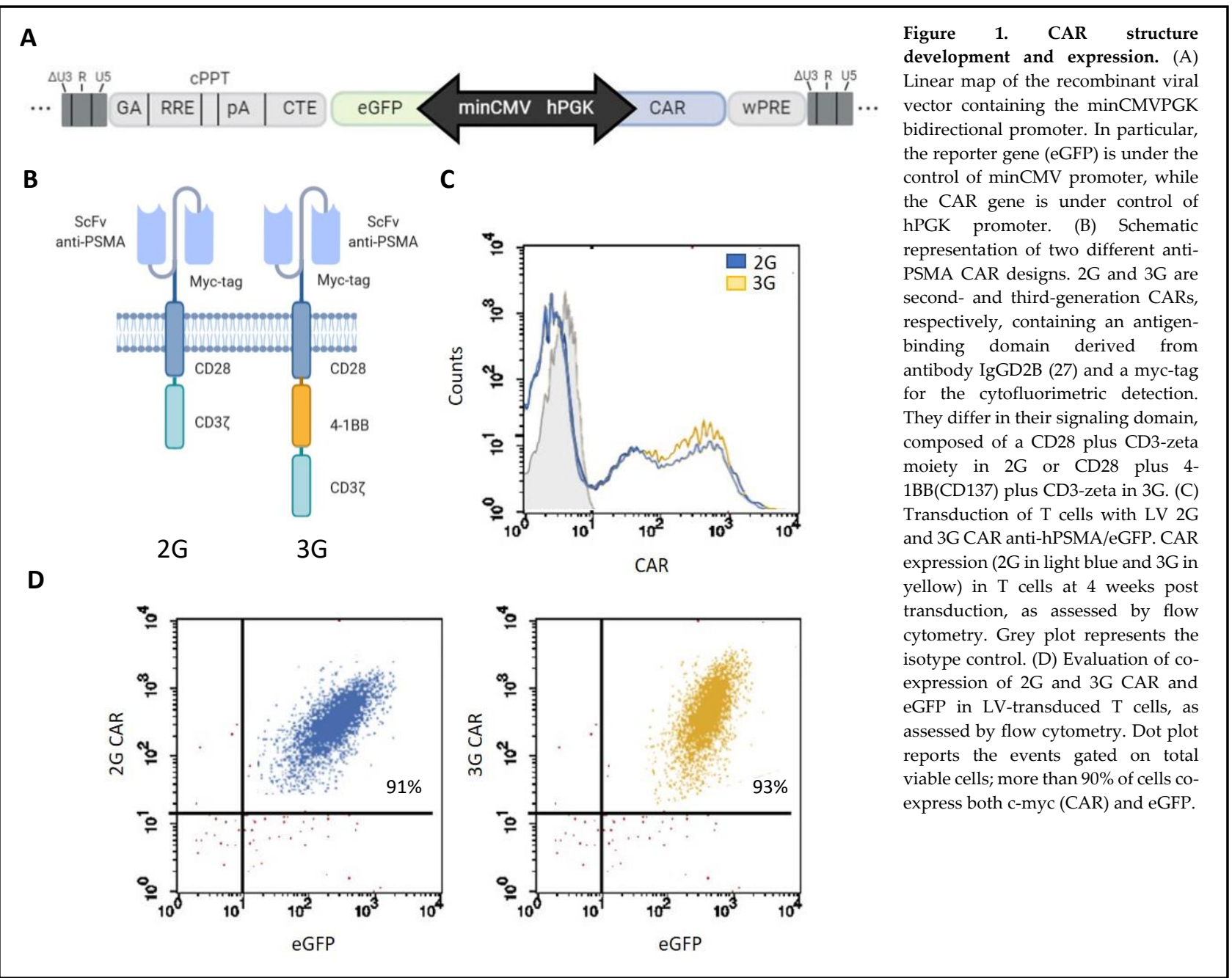

3.2. $2 G$ and $3 G$ CAR T cells show a similar expansion of the CAR-expressing population, but $3 G$ population quickly displays a more differentiated effector memory phenotype

For the generation of $T$ cell populations expressing the 2G or the 3G CAR anti-PSMA, we used a previously described [25] rapid expansion protocol that involve weekly re-stimulation with PC3PSMA cells that allowed a rapid and sustained proliferation of CAR-T cells. In both generations, the subsequent encounter with the antigen led to the expansion of the CAR-expressing population (Fig.2A, left panel). Moreover, to better define the state of differentiation of CAR-transduced T lymphocytes in the post-infection period during antigenic re-stimulations, expression of different surface markers (CD62L, CD27, CD28, CCR7, CD57) was assessed by cytofluorimetric analysis. One week after transduction, the emerging profile of $2 \mathrm{G}$ CAR $T$ was essentially of 
A
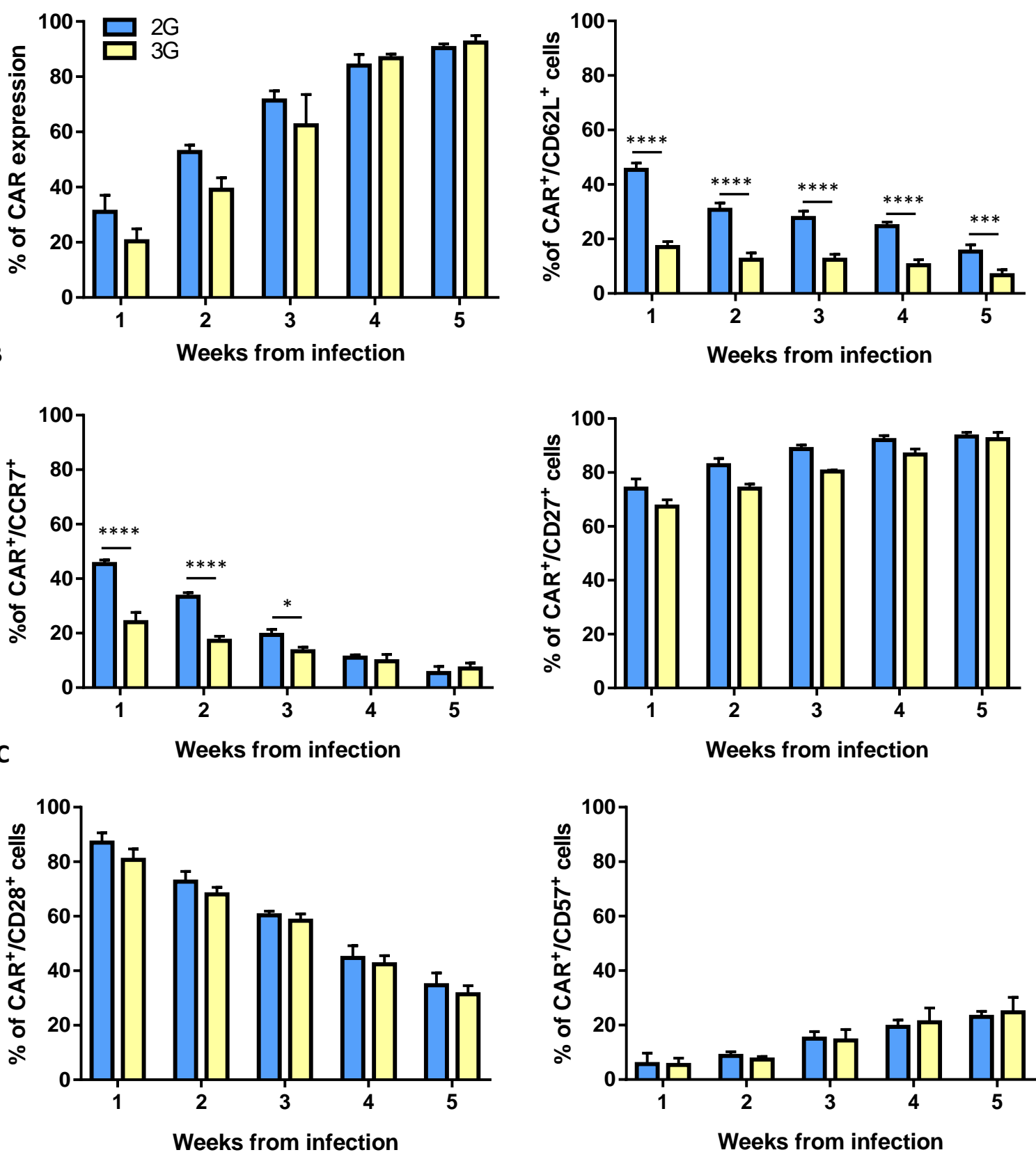

Figure 2. Differentiation pattern of the 2G and 3G CAR-expressing populations. (A) Left panel, $2 \mathrm{G}$ and $3 G$ CAR expression in transduced T cells upon stimulation. Istograms report percentage of $2 \mathrm{G}$ (light blue) and $3 \mathrm{G}$ (yellow) CAR ${ }^{+}$cells in LV CAR hPSMA/eGFP transduced T cell populations at different time points post transduction. Right panel, expression of CD62L. (B) Left panel, expression of CCR7, and in right panel, expression of CD27. (C) Left panel, expression of $C D 28$, and in right panel, expression of $C D 57$. All surface markers were evaluated in $2 \mathrm{G}$ and $3 G$ CAR-expressing populations at different time points post transduction, as assessed by flow cytometry. Figures show mean $+/-2$ SD of at least three independent experiments. ${ }^{*} p<0.05,{ }^{* *} p<0.01$, $* * * p<0.001^{* * * *} p<0.0001$ by two-way ANOVA.

early effector T cells, as shown by the high expression of CD62L (Fig.2A, right panel), CD27 (Fig.2B, right panel) and CD28 (Fig.2C, left panel), the presence of CCR7 (Fig.2B, left panel) positive cells and the low expression of CD57 (Fig.2C, right panel). Instead, 3G CAR T cells show, already at the first week post transduction, a significant difference in the expression of CD62L and CCR7, displaying very quickly a more differentiated effector memory phenotype. Following re-stimulations with the antigen, both $\mathrm{T}$ cells populations acquired an intermediate effector memory phenotype with the progressive down-modulation of CD62L, CCR7, CD28 and a slight increase in CD57 expression. 


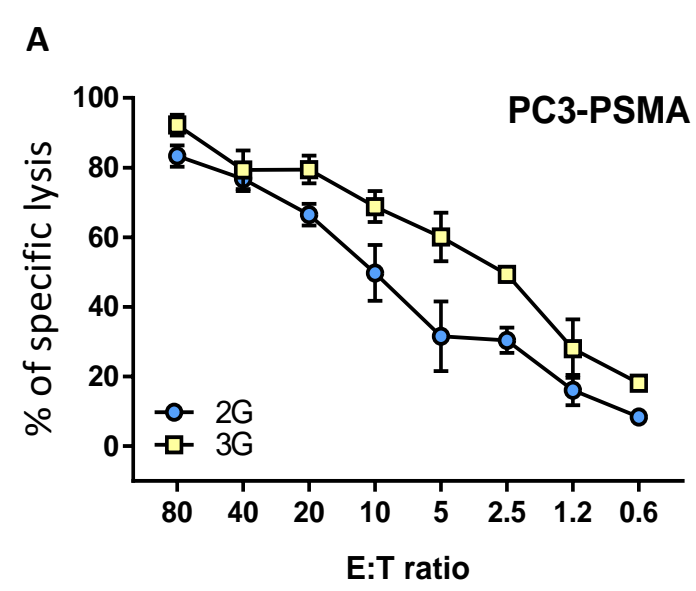

B
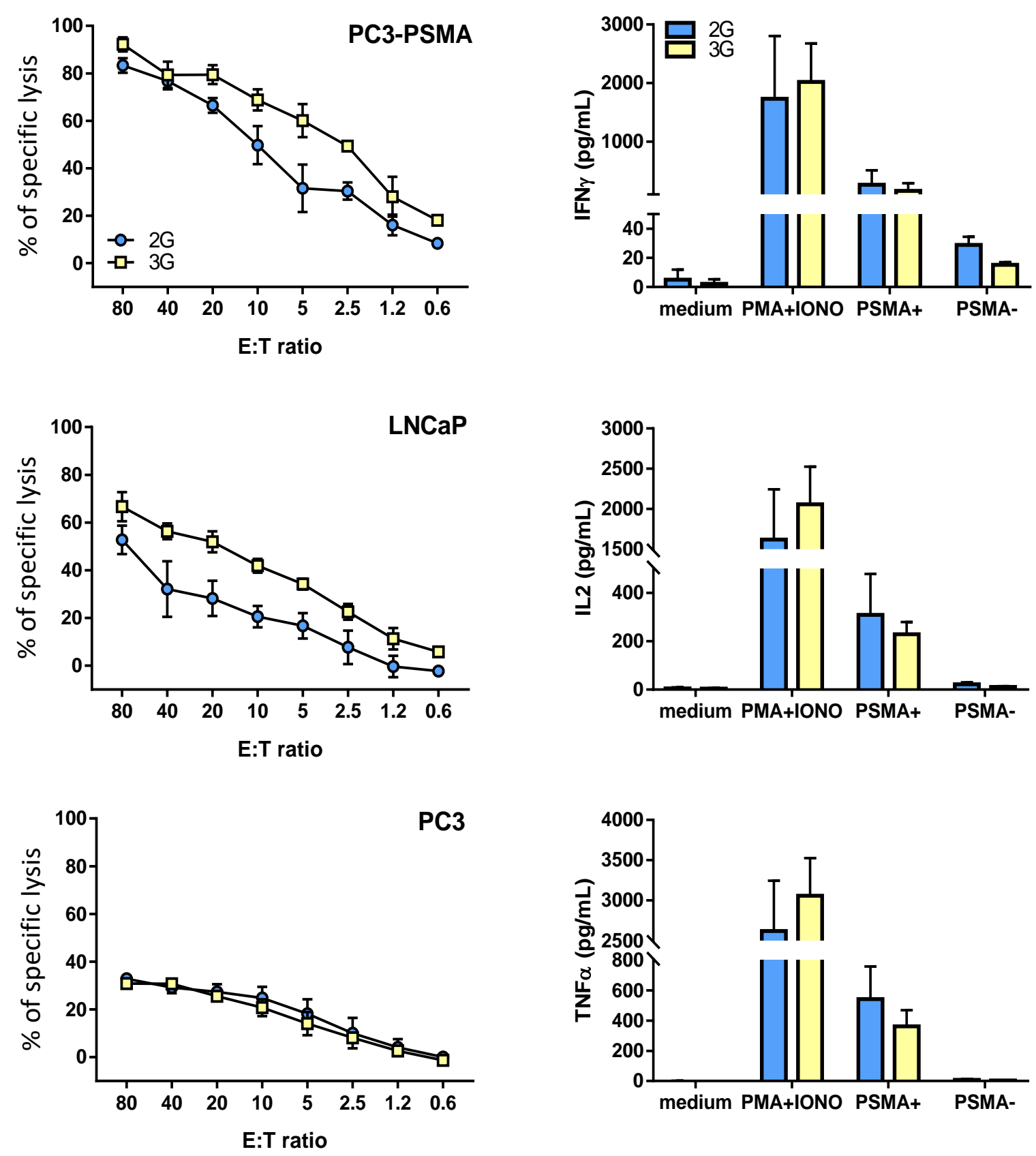

Figure 3. Functional characterization of the 2 G and $3 G$ CAR-expressing populations. (A) Lytic activity of the 2G (light blue) and $3 G$ CAR (yellow)-expressing populations. Cytotoxicity was analyzed at 15 days post-transduction; PC3-PSMA, LNCaP and PC3, cells were used as target cells. (B) Cytokine release upon antigen stimulation. Upper panel: IFN- $\gamma$, middle panel: IL-2 and lower panel: TNF- $\alpha$. The cytokine production was analyzed at different time points after T cells transduction by stimulating 2 G and 3G CAR populations with PC3-PSMA or PC3 cancer cell lines. 2G and 3G CAR T cells unstimulated or treated with $\mathrm{PMA} /$ Ionomycin represented the negative and positive controls, respectively. Figures show mean $+/ 2$ SD of 3 independent experiments.

3.3. 2G and 3G CAR T cells show comparable tumor cell killing and cytokine production following exposure to PSMA+ cells 
CAR expression provided the transduced population with the ability to recognize the PSMA antigen on the surface of prostate tumor cell lines, and to mediate a high and specific cytotoxicity. Indeed, both the 2G and 3G CAR populations lysed the PSMA-transfected PC3 cells (Fig.3A, upper panel) while sparing the antigen-negative counterpart (Fig.3A, lower panel); more importantly, they were also capable of recognizing the LNCaP target cells that naturally harbor the PSMA antigen (Fig. 3A, middle panel). Other than exerting a relevant cytotoxic activity, both generations of CAR-transduced T cells also produced high and comparable levels of IFN- $\gamma$, IL-2 and TNF- $\alpha$ in response to PSMAexpressing tumor cells, but not against PSMA negative control cells (Fig. 3B).

\subsection{G CAR T cells become exhausted during in vitro expansion}

After an initial rapid and sustained proliferation of CAR T cells, 4 weeks from transduction in 3G CAR T cell population a slight slowdown of proliferation set in, with a significant difference in the number of total CAR T cells from day 25 to day 40 ( $\mathrm{p}<0.0001)$ after transduction (Fig.4A, left panel). By day 25, CD28- $\zeta-4-1 \mathrm{BB}$ (3G) CAR T cells showed a cell surface profile consistent with exhaustion, including higher and significant difference in expression of PD-1 $(\mathrm{p}<0.0001)$, TIM-3 $(\mathrm{p}=0.0019)$ and LAG-3 ( $p=0.0031$ ) compared to 2G CAR T cell population (Fig 4A, right panel). 3G CAR T cells expanded less efficiently and showed higher rates of apoptosis (Ann $\mathrm{V}^{+} ; \mathrm{p}<0.0031$ ), compared to the 2 G CAR T cell population, more probably induced by the FAS-FASL pathway (FASL $; \mathrm{p}=0.0454$ ) (Fig 4B, left panel). Furthermore, 3G CAR T cells poorly produced IL-2, TNF- $\alpha$ and IFN- $\gamma$, compared with $2 \mathrm{G}$ CAR T cells following exposure to $\mathrm{PSMA}^{+}$cells. Together, these phenotypic and functional studies demonstrate that $3 \mathrm{G}$ CAR T cells become rapidly exhausted during in vitro culture, whereas similar effects do not occur in 2G CAR T, cells stimulated in the same manner. More importantly, this exhaustion causes the impossibility to expand the 3G-CAR cells sufficiently to obtain the minimum number of cells requested for the in vivo therapies 
A
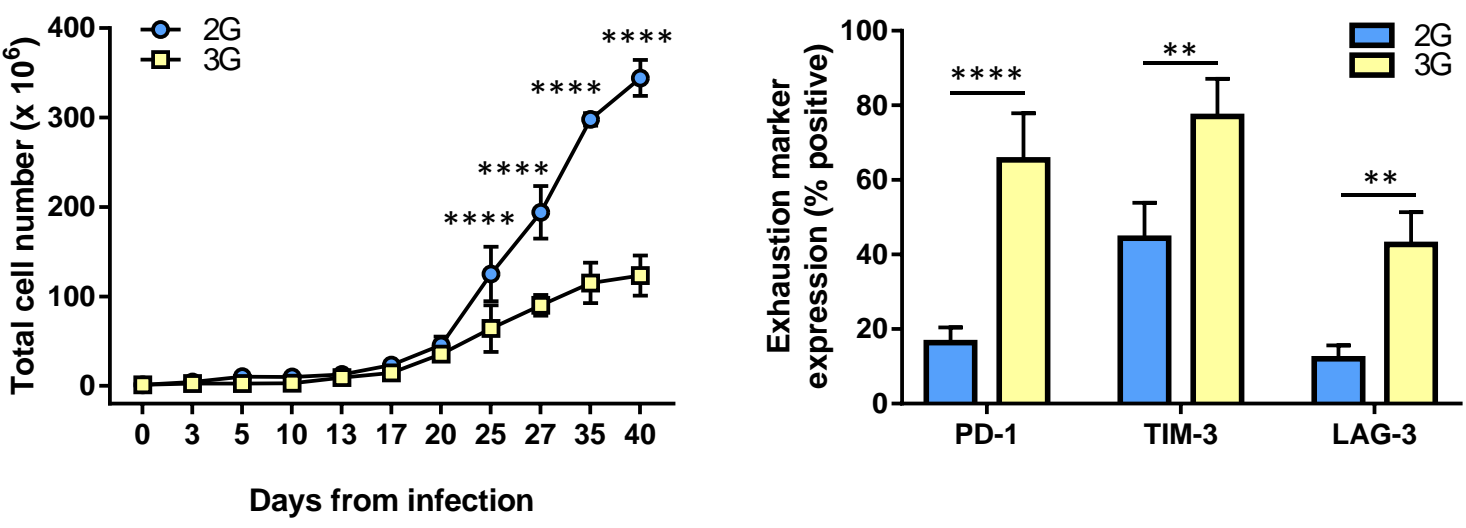

B
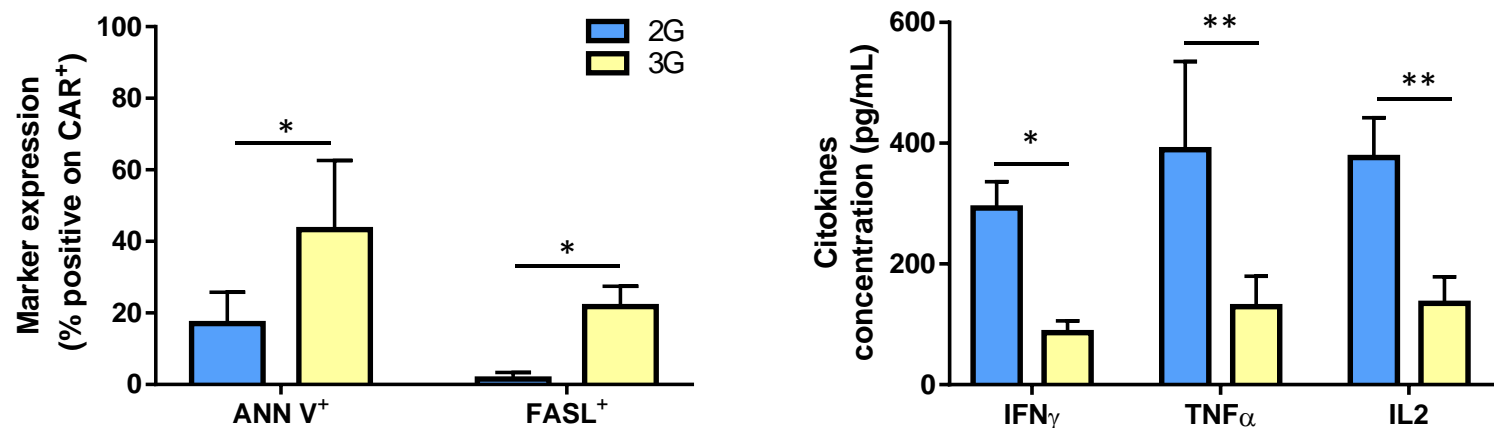

Figure 4. 3G CAR T cells become exhausted during in vitro expansion. (A) Left panel, expansion of the 2G (light blue) and 3G CAR (yellow)-expressing populations upon weekly antigen stimulation. Right panel, quantification of exhaustion marker expression at 25-35 days from transduction. (B) Left panel, quantification of $A N N V^{+}$and $\mathrm{FASL}^{+}$in $2 \mathrm{G}$ and $3 \mathrm{G}$ CAR populations at 25-35 days from transduction. Right panel, cytokines production upon stimulation with PC3-PSMA target cells at $25-35$ days from transduction. Figures show mean $+/ 2$ SD of 3 independent experiments. $* p<0.05, * * p<0.01$, $* * * p<0.001 * * * * p<0.0001$ by two way ANOVA.

\section{Discussion}

Impressive early clinical results were reported following administration of CD19 CAR T cells and demonstrate that CAR therapies represents one of the most effective immune-based treatments of cancer $[27,28]$. Conversely, the difficulties intrinsic to the treatment of solid tumors (in particular, the choice of suitable and safe target antigens) and the largely unsatisfactory results obtained thus far make them a less appealing but still open battle field for immunotherapeutic strategies. Among solid tumors, PCa is the most common tumor between males in Europe and the US [29]. The specific targeting of this tumor by ACT represents a promising strategy to control the disease, as PCa expresses distinct surface tumor-associated antigens that can be exploited for immune interventions. Among these, PSMA is a well-known tumor-associated antigen, and its expression and enzymatic activity are increased in PCa compared to normal prostate epithelium, thereby making PSMA an ideal target for ACT. In our laboratory, we have already described a very efficient CAR against PSMA [25] based on an high-affinity specific mAb [26]. Nevertheless, important lessons have been learned from trials, particularly with regard to data concerning the improved persistence and efficacy of CAR T cells, as well as the importance of enhancing co-stimulation and reducing T-cell exhaustion [30]. It remains furthermore unclear to what extent this relates to variable potencies of the chimeric receptors themselves. To this purpose, in this study we have generated two tumor antigen-specific receptors that signal, through serial endodomains, a second generation (2G) CAR 
bearing both $\mathrm{CD} 28$ and $\mathrm{CD} 3 \zeta$ signaling domains and a 3rd generation (3G) CAR combining CD28 and $\mathrm{CD} 3 \zeta$ with a $4-1 \mathrm{BB}$ co-stimulatory domain. Their function was analyzed in comparative fashion in cultured human primary T lymphocytes. Both chimeric receptors effectively killed PSMA+tumor cell lines in vitro, underscoring their antigen-specificity and sufficient signaling strength to trigger cytolysis. However, already in the first week post transduction, 3G CAR T cell population has shown a more differentiated effector phenotype that is more prone to AICD compared to the early effector $2 \mathrm{G}$ population. Moreover, in proliferative assays we observed that the expansion rate of these two types of CAR $\mathrm{T}$ cells was superimposable during the first three weeks of culture. Thereafter, 2G CAR displays an expansion rate, whereas 3G CAR T cells reached a plateau and then started to die. Here, we provide the unexpected observation that PSMA CARs incorporating an highaffinity scFv derived from the well-studied D2B antibody [26] and CD28-4-1BB produce a "superstimulation" that accelerates apoptosis and exhaustion of 3G CAR T population during in vitro expansion. This exhaustion is characterized by high expression of exhaustion markers, poor proliferative capacity, and poor cytokine production. These observations provide the rationale to assert that co-stimulatory signals could induce an activation-induced cell death (AICD) as a result of augmented FasL expression and, more importantly, causing the impossibility to expand the 3G CAR cells sufficiently enough to obtain the minimum number of cells requested for in vivo therapies. The observation that some CARs lead to impaired $\mathrm{T}$ cell function and exhaustion illustrates the importance of optimizing CAR engineering and a need for enhanced understanding of how receptor structure impacts on function. The role of T-cell exhaustion is particularly relevant in the context of adoptive T-cell therapy for treatment of solid tumors, since it has been clearly shown that expansion and persistence of the adoptively transferred cells are crucial for patient outcome [30-32]. The possibility to modulate the degree of T-cell exhaustion, which limits anti-tumor efficacy, remains largely unexplored. Our work contributes to a growing literature demonstrating that structural characteristics of CAR endodomains can critically impact on the functionality of these novel receptors $[15,20,22,23,33]$. In this work, where we used an identical high affinity anti-PSMA scFvs with a CD28 or a combined CD28-4-1BB co-stimulatory domain, we have demonstrated that the presence of a high-affinity $\mathrm{scFv}$ in addition to a two-costimulatory molecule most likely produces an overloading of stimulation, engendering an exhaustion profile of the cells that stop proliferating. Given these findings, we demonstrate as other groups [19] that second generation CAR-T cells outperform their third generation counterparts. A similar phenomenon was described by Hombachet et al., who described that cytokine-induced killer (CIK) cells expressing a second generation $\mathrm{CAR}$ containing $\mathrm{CD} 28-\mathrm{CD} 3 \zeta$ domains presented higher antitumor efficacy than CIK expressing a third generation CD28-OX40-CD3 $\zeta$ CAR. In that setting, as in ours, the detrimental effect of the additional co-stimulatory domain was attributed to increased activation-induced cell death in CIK cells expressing the third generation CAR [20]. On the other hand, other authors postulate that third-generation CARs containing both CD28and 4-1BB outperform secondgeneration CARs containing only CD28, as a general rule [22,34]. The discrepancy between our results and previously published reports imply that the optimal CAR design might need to be empirically determined for each individual target and for each $\mathrm{scFv}$, because too much stimulation could be detrimental.

\section{Abbreviations}

${ }^{51} \mathrm{Cr}$, chromium-51; 2G-CAR, second-generation CAR; 3G-CAR, third-generation CAR; ACT, adoptive cancer immunotherapy; AICD, activation-induced cell death; ATCC, American Type Culture Collection; Alpha MEM, minimum essential medium eagle, alpha modification; CAR-T, chimeric antigen receptor T; DMEM, Dulbecco's Modified Eagle Medium; E/T, Effector/Target; eGFP, enhanced green gluorescent protein; FBS, Fetal Bovine Serum; IFN- $\gamma$, interferon- $\gamma$; ICD, intracellular costimulatory domain; LV, lentiviral transfer 
vector; mAb, monoclonal antibody;PBS, phosphate buffered saline; PCa, prostate cancer; PMA, phorbolmyristate-acetate; PSMA, human prostate-specific membrane antigen; RPMI, Roswell Park Memorial Institute; $\mathrm{ScFV}$, single-chain variable fragment; SD, standard deviation; TME, tumor microenvironment; TMD,transmembrane domain.

Authors' contributions: Conceived and designed the experiments: G.Z. and A.R.; Performed the experiments: G.Z., I.M.M., Analyzed the data: D.C., A.T; Contributed reagents/materials/analysis tools: G.F.; Contributed to the writing of the manuscript: A.R., G.Z.

Funding: The research leading to these results has received funding from Fondazione AIRC under IG 2018-ID. 21354 project - P.I. Rosato Antonio; 5 per Mille 2019 - ID. 22759 program - P.I. Piccolo Stefano, G.L. Rosato Antonio; BIRD180331/18 from University of Padova to AR, and the Ministry of Health-Alliance Against Cancer (MoH-ACC) project "Research project on CAR T cells for hematological malignancies and solid tumors" to AR.

Conflicts of Interest: The authors declare no conflict of interest.

\section{References}

1. Maher, J. Clinical Immunotherapy of B-Cell Malignancy Using CD19-Targeted CAR T-Cells. Curr. Gene Ther. 2014, 14, 35-43, doi:10.2174/1566523213666131223130554.

2. Davila, M.L.; Brentjens, R.J. CD19-Targeted CAR T cells as novel cancer immunotherapy for relapsed or refractory B-cell acute lymphoblastic leukemia. Clin. Adv. Hematol. Oncol. 2016, $14,802-808$.

3. Kochenderfer, J.N.; Dudley, M.E.; Kassim, S.H.; Somerville, R.P.T.; Carpenter, R.O.; StetlerStevenson, M.; Yang, J.C.; Phan, G.Q.; Hughes, M.S.; Sherry, R.M.; et al. Chemotherapyrefractory diffuse large B-cell lymphoma and indolent B-cell malignancies can be effectively treated with autologous T cells expressing an anti-CD19 chimeric antigen receptor. J. Clin. Oncol. 2015, 33, 540-9, doi:10.1200/JCO.2014.56.2025.

4. Scarfò, I.; Maus, M. V. Current approaches to increase CAR T cell potency in solid tumors: targeting the tumor microenvironment. J. Immunother. Cancer 2017, 5, 28, doi:10.1186/s40425017-0230-9.

5. Newick, K.; Moon, E.; Albelda, S.M. Chimeric antigen receptor T-cell therapy for solid tumors. Mol. Ther. - Oncolytics 2016, 3, 16006, doi:10.1038/mto.2016.6.

6. Gilham, D.E.; Maher, J. 'Atypical' CAR T cells: NKG2D and Erb-B as examples of natural receptor/ligands to target recalcitrant solid tumors. Immunotherapy 2017, 9, 723-733, doi:10.2217/imt-2017-0045.

7. Haberkorn, U.; Eder, M.; Kopka, K.; Babich, J.W.; Eisenhut, M. New strategies in prostate cancer: Prostate-specific membrane antigen (PSMA) ligands for diagnosis and therapy. Clin. Cancer Res. 2016, doi:10.1158/1078-0432.CCR-15-0820.

8. Lizee, G.; Cantu, M.A.; Hwu, P. Less Yin, More Yang: Confronting the Barriers to Cancer Immunotherapy. Clin. Cancer Res. 2007, 13, 5250-5255, doi:10.1158/1078-0432.CCR-07-1722.

9. Rossig, C.; Bollard, C.M.; Nuchtern, J.G.; Rooney, C.M.; Brenner, M.K. Epstein-Barr virusspecific human T lymphocytes expressing antitumor chimeric T-cell receptors: potential for improved immunotherapy. Blood 2002, 99, 2009-2016, doi:10.1182/blood.V99.6.2009.

10. Shrikant, P.; Mescher, M.F. Control of syngeneic tumor growth by activation of CD8+ T cells: efficacy is limited by migration away from the site and induction of 
nonresponsiveness. J. Immunol. 1999, 162, 2858-66.

11. Robbins, P.F.; Dudley, M.E.; Wunderlich, J.; El-Gamil, M.; Li, Y.F.; Zhou, J.; Huang, J.; Powell, D.J.; Rosenberg, S.A. Cutting Edge: Persistence of Transferred Lymphocyte Clonotypes Correlates with Cancer Regression in Patients Receiving Cell Transfer Therapy. J. Immunol. 2004, 173, 7125-7130, doi:10.4049/jimmunol.173.12.7125.

12. Kowolik, C.M.; Topp, M.S.; Gonzalez, S.; Pfeiffer, T.; Olivares, S.; Gonzalez, N.; Smith, D.D.; Forman, S.J.; Jensen, M.C.; Cooper, L.J.N. CD28 costimulation provided through a CD19specific chimeric antigen receptor enhances in vivo persistence and antitumor efficacy of adoptively transferred T cells. Cancer Res. 2006, 66, 10995-1004, doi:10.1158/0008-5472.CAN06-0160.

13. Milone, M.C.; Fish, J.D.; Carpenito, C.; Carroll, R.G.; Binder, G.K.; Teachey, D.; Samanta, M.; Lakhal, M.; Gloss, B.; Danet-Desnoyers, G.; et al. Chimeric receptors containing CD137 signal transduction domains mediate enhanced survival of $\mathrm{T}$ cells and increased antileukemic efficacy in vivo. Mol. Ther. 2009, 17, 1453-64, doi:10.1038/mt.2009.83.

14. Haso, W.; Lee, D.W.; Shah, N.N.; Stetler-Stevenson, M.; Yuan, C.M.; Pastan, I.H.; Dimitrov, D.S.; Morgan, R.A.; FitzGerald, D.J.; Barrett, D.M.; et al. Anti-CD22-chimeric antigen receptors targeting B-cell precursor acute lymphoblastic leukemia. Blood 2013, 121, 1165-74, doi:10.1182/blood-2012-06-438002.

15. Zhao, Z.; Condomines, M.; van der Stegen, S.J.C.; Perna, F.; Kloss, C.C.; Gunset, G.; Plotkin, J.; Sadelain, M. Structural Design of Engineered Costimulation Determines Tumor Rejection Kinetics and Persistence of CAR T Cells. Cancer Cell 2015, 28, 415-428, doi:10.1016/j.ccell.2015.09.004.

16. van der Stegen, S.J.C.; Hamieh, M.; Sadelain, M. The pharmacology of second-generation chimeric antigen receptors. Nat. Rev. Drug Discov. 2015, 14, 499-509, doi:10.1038/nrd4597.

17. Hombach, A.; Holzinger, A.; Abken, H. The Weal and Woe of Costimulation in the Adoptive Therapy of Cancer with Chimeric Antigen Receptor (CAR)-Redirected T Cells. Curr. Mol. Med. 2013, 13, 1079-1088, doi:10.2174/1566524011313070003.

18. Hombach, A.A.; Abken, H. Costimulation by chimeric antigen receptors revisited the T cell antitumor response benefits from combined CD28-OX40 signalling. Int. J. Cancer 2011, 129, 2935-2944, doi:10.1002/ijc.25960.

19. Abate-Daga, D.; Lagisetty, K.H.; Tran, E.; Zheng, Z.; Gattinoni, L.; Yu, Z.; Burns, W.R.; Miermont, A.M.; Teper, Y.; Rudloff, U.; et al. A Novel Chimeric Antigen Receptor Against Prostate Stem Cell Antigen Mediates Tumor Destruction in a Humanized Mouse Model of Pancreatic Cancer. Hum. Gene Ther. 2014, 25, 1003-1012, doi:10.1089/hum.2013.209.

20. Hombach, A.A.; Rappl, G.; Abken, H. Arming Cytokine-induced Killer Cells With Chimeric Antigen Receptors: CD28 Outperforms Combined CD28-OX40 “Super-stimulation.” Mol. Ther. 2013, 21, 2268-2277, doi:10.1038/mt.2013.192.

21. Long, A.H.; Haso, W.M.; Shern, J.F.; Wanhainen, K.M.; Murgai, M.; Ingaramo, M.; Smith, J.P.; Walker, A.J.; Kohler, M.E.; Venkateshwara, V.R.; et al. 4-1BB costimulation ameliorates $\mathrm{T}$ cell exhaustion induced by tonic signaling of chimeric antigen receptors. Nat. Med. 2015, 21, 581-590, doi:10.1038/nm.3838. 
22. Zhong, X.-S.; Matsushita, M.; Plotkin, J.; Riviere, I.; Sadelain, M. Chimeric Antigen Receptors Combining 4-1BB and CD28 Signaling Domains Augment PI3kinase/AKT/Bcl-XL Activation and CD8+ T Cell-mediated Tumor Eradication. Mol. Ther. 2010, 18, 413-420, doi:10.1038/mt.2009.210.

23. Guedan, S.; Posey, A.D.; Shaw, C.; Wing, A.; Da, T.; Patel, P.R.; McGettigan, S.E.; CasadoMedrano, V.; Kawalekar, O.U.; Uribe-Herranz, M.; et al. Enhancing CAR T cell persistence through ICOS and 4-1BB costimulation. JCI Insight 2018, 3, doi:10.1172/jci.insight.96976.

24. Ghosh, A.; Wang, X.; Klein, E.; Heston, W.D.W. Novel role of prostate-specific membrane antigen in suppressing prostate cancer invasiveness. Cancer Res. 2005, 65, 727-31.

25. Zuccolotto, G.; Fracasso, G.; Merlo, A.; Montagner, I.M.; Rondina, M.; Bobisse, S.; Figini, M.; Cingarlini, S.; Colombatti, M.; Zanovello, P.; et al. PSMA-Specific CAR-Engineered T Cells Eradicate Disseminated Prostate Cancer in Preclinical Models. PLoS One 2014, doi:10.1371/journal.pone.0109427.

26. Frigerio, B.; Fracasso, G.; Luison, E.; Cingarlini, S.; Mortarino, M.; Coliva, A.; Seregni, E.; Bombardieri, E.; Zuccolotto, G.; Rosato, A.; et al. A single-chain fragment against prostate specific membrane antigen as a tool to build theranostic reagents for prostate cancer. Eur. J. Cancer 2013, 49, 2223-2232, doi:10.1016/j.ejca.2013.01.024.

27. Lee, D.W.; Kochenderfer, J.N.; Stetler-Stevenson, M.; Cui, Y.K.; Delbrook, C.; Feldman, S.A.; Fry, T.J.; Orentas, R.; Sabatino, M.; Shah, N.N.; et al. T cells expressing CD19 chimeric antigen receptors for acute lymphoblastic leukaemia in children and young adults: a phase 1 dose-escalation trial. Lancet (London, England) 2015, 385, 517-528, doi:10.1016/S01406736(14)61403-3.

28. Maude, S.L.; Teachey, D.T.; Porter, D.L.; Grupp, S.A. CD19-targeted chimeric antigen receptor T-cell therapy for acute lymphoblastic leukemia. Blood 2015, 125, 4017-4023, doi:10.1182/blood-2014-12-580068.

29. Miller, K.D.; Nogueira, L.; Mariotto, A.B.; Rowland, J.H.; Yabroff, K.R.; Alfano, C.M.; Jemal, A.; Kramer, J.L.; Siegel, R.L. Cancer treatment and survivorship statistics, 2019. CA. Cancer J. Clin. 2019, doi:10.3322/caac.21565.

30. Caruana, I.; Weber, G.; Ballard, B.C.; Wood, M.S.; Savoldo, B.; Dotti, G. K562-Derived Whole-Cell Vaccine Enhances Antitumor Responses of CAR-Redirected Virus-Specific Cytotoxic T Lymphocytes In Vivo. Clin. Cancer Res. 2015, 21, 2952-62, doi:10.1158/10780432.CCR-14-2998.

31. Caruana, I.; Savoldo, B.; Hoyos, V.; Weber, G.; Liu, H.; Kim, E.S.; Ittmann, M.M.; Marchetti, D.; Dotti, G. Heparanase promotes tumor infiltration and antitumor activity of CARredirected T lymphocytes. Nat. Med. 2015, 21, 524-9, doi:10.1038/nm.3833.

32. Porter, D.L.; Hwang, W.-T.; Frey, N. V; Lacey, S.F.; Shaw, P.A.; Loren, A.W.; Bagg, A.; Marcucci, K.T.; Shen, A.; Gonzalez, V.; et al. Chimeric antigen receptor T cells persist and induce sustained remissions in relapsed refractory chronic lymphocytic leukemia. Sci. Transl. Med. 2015, 7, 303ra139, doi:10.1126/scitranslmed.aac5415.

33. Hudecek, M.; Sommermeyer, D.; Kosasih, P.L.; Silva-Benedict, A.; Liu, L.; Rader, C.; Jensen, M.C.; Riddell, S.R. The Nonsignaling Extracellular Spacer Domain of Chimeric Antigen Receptors Is Decisive for In Vivo Antitumor Activity. Cancer Immunol. Res. 2015, 3, 125-135, 
doi:10.1158/2326-6066.CIR-14-0127.

34. Tammana, S.; Huang, X.; Wong, M.; Milone, M.C.; Ma, L.; Levine, B.L.; June, C.H.; Wagner, J.E.; Blazar, B.R.; Zhou, X. 4-1BB and CD28 signaling plays a synergistic role in redirecting umbilical cord blood T cells against B-cell malignancies. Hum. Gene Ther. 2010, 21, 75-86, doi:10.1089/hum.2009.122. 